\title{
Branches of index-preserving solutions to systems of second order ODEs
}

\author{
Francesca Dalbono
}

\begin{abstract}
We investigate the existence of a continuum of index-preserving solutions to a Dirichlet problem associated with a parameter-dependent system of second order ordinary differential equations, developing a detailed analysis on the behaviour of the branches of nontrivial solutions. Our approach is based on the Rabinowitz global bifurcation Theorem combined with the notion of index and nullity of suitable linear boundary value problems. An application of the result to the study of branches of odd, periodic solutions for suitable systems of two linearly coupled pendulums of lenghts variables is also analyzed.
\end{abstract}

Mathematics Subject Classification (2000). 34B15 (34C25).

Keywords. Second order systems, Index-preserving solutions, Bifurcation.

\section{Introduction and statement of the main result}

This paper is devoted to the study of the bifurcation of a continuum of solutions for the parameter-dependent problem

$$
\left\{\begin{array}{l}
u^{\prime \prime}(t)+A(t, u(t), \lambda) u(t)=0 \\
u(0)=0=u(T),
\end{array}\right.
$$

where $T>0$ is fixed. Denoting by $M_{s}(n)$ the set of $n \times n$ real symmetric matrices, we assume that $A:[0, T] \times \mathbb{R}^{n} \times \mathbb{R} \rightarrow M_{s}(n)$ is of the form

$$
A(t, x, \lambda)=L(t)+\lambda H(t)+R(t, x, \lambda),
$$

The work has been performed in the frame of the Italian M.I.U.R. project "Variational and topological methods in the study of nonlinear phenomena" and of the Italian GNAMPA-I.N.d.A.M. project "Nonlinear analysis techniques for boundary value problems associated to differential equations". 
where $L, H \in C\left([0, T] ; M_{s}(n)\right)$ and $R:[0, T] \times \mathbb{R}^{n} \times \mathbb{R} \rightarrow M_{s}(n)$ is a continuous function satisfying $R(t, x, \lambda) x=o(\|x\|)$ when $x \rightarrow 0, x \in \mathbb{R}^{n}$, uniformly on $t \in[0, T]$ and on bounded $\lambda$ intervals.

We are concerned with the linearization at $u=0$ of system (1.1) and we require $\lambda=1$ to be an eigenvalue of the linearized system. We focus our attention on the continuum bifurcating from $(\lambda, u(\cdot)) \equiv(1,0)$. For simplicity, we have chosen to bifurcate from the eigenvalue $\lambda=1$. Note that we could have bifurcated from any other eigenvalue, as observed in Remark 2.8.

Our work is based on the Rabinowitz global bifurcation theorem [23] combined with the notion and the properties of the index and nullity associated with a suitable linear boundary value problem. In order to introduce the definitions of index and of nullity of a path of symmetric matrices (see [8]), let us recall the following proposition (cf., for instance, [8])

Proposition 1.1. For every $K \in L^{\infty}\left([0, T] ; M_{s}(n)\right)$, the problem

$$
\begin{aligned}
& u^{\prime \prime}(t)+(K(t)+\eta \mathrm{Id}) u(t)=0 \\
& u(0)=0=u(T)
\end{aligned}
$$

admits a sequence of eigenvalues $\eta_{1}(K) \leq \eta_{2}(K) \leq \cdots \leq \eta_{j}(K) \rightarrow+\infty$ as $j \rightarrow+\infty$ such that the eigenspace associated with each eigenvalue has dimension one.

Moreover $H_{0}^{1}\left([0, T] ; \mathbb{R}^{n}\right):=\left\{x:[0, T] \rightarrow \mathbb{R}^{n} \mid x(\cdot)\right.$ is continuous on $[0, T]$, satisfies $x(0)=0=x(T)$, and $\left.x^{\prime} \in L^{2}\left([0, T] ; \mathbb{R}^{n}\right)\right\}$ admits a basis of eigenvectors of $K$.

Definition 1.2. Given $K \in L^{\infty}\left([0, T] ; M_{s}(n)\right)$, its index $i(K)$ is defined as the number of negative eigenvalues of problem (1.3), and its nullity $\nu(K)$ as the number of zero eigenvalues of problem (1.3).

We remark that the index of $K \in L^{\infty}\left([0, T] ; M_{s}(n)\right)$ above introduced coincides, in the non-degenerate case, with the Morse index of the boundary value problem

$$
u^{\prime \prime}(t)+K(t) u(t)=0, \quad u(0)=0=u(T) .
$$

Notice that $\nu(K) \neq 0$ if and only if there exists a nontrivial solution of (1.4).

Throughtout the paper, we assume that $\nu(L+H) \neq 0$ (cf. (1.10) below), which, according to the previous remark and to the fact that $A(\cdot, 0,1)=L+H$, implies that $\lambda=1$ is an eigenvalue of the linearized system.

We study the case in which $A(\cdot, x, \lambda)$ is bounded from above by a path of symmetric matrices $B(\cdot)$, having index equal to 1 (cf. assumptions (1.8) and (1.9) below). This kind of assumption guarantees the simplicity of the eigenvalue $\lambda=1$ from which we are interested in bifurcating and allows us to apply the Rabinowitz global bifurcation theorem [23]. An analogous situation occurs when we assume the existence of lower and upper bounds on $A(\cdot, x, \lambda)$, represented by two paths of symmetric matrices whose indexes are two consecutive natural numbers (cf. hypotheses (2.12) and (2.13) below). 
Our idea of handling nonlinearities which are bounded from above and below by two paths of symmetric matrices with respective different indexes originates from the paper [17], dealing with existence and uniqueness of periodic solutions of the system

$$
u^{\prime \prime}+\nabla G(u)=p(t, u) .
$$

In particular, in [17] Lazer shows that the problem $u^{\prime \prime}(t)+\nabla G(u)=0$ does not admit any nontrivial $2 \pi$-periodic solution whenever $\nabla G(0)=0$ and the Hessian matrix of $G$ is bounded from above and from below by suitable constant symmetric matrices having the same index. Inspired by this result, we have decided to relax the Lazer's condition in order to obtain (instead of an uniqueness theorem) the existence of branches of nontrivial solutions for our parameter-dependent problem. To this aim, we have decided to allow one jump between the lower bound and the upper bound indexes. We refer to the first part of Sect. 4 for the statement of Lazer's result (cf. Theorems 4.1 and 4.2), for its generalization to the case of nonconstant bounds in a Dirichlet boundary conditions setting (cf. Theorem 4.4, which is an immediate consequence of the known index comparison properties), and for more detailed comments on this subject.

We also wish to mention the papers $[1,18,25]$, written in the same spirit of the Lazer's paper [17], requiring index-preserving upper and lower bounds on the Hessian matrix of $G$ in order to achieve periodic solutions of (1.5).

Another interesting multiplicity result regarding periodic systems of the form $u^{\prime \prime}+L(t) u+\nabla G(t, u)=0$ has been established in [22], when the index and nullity of $L$ are, respectively, equal to 0 and 1 . This resonance condition recalls the setting of Theorem 1.3 and Proposition 2.2 below.

Our assumptions involving estimates on the nonlinearity combined with the hypothesis concerning the non zero nullity of $L+H$ lead to the existence of a continuum of solutions to (1.1). If, in addition, we impose the positive definiteness of the path of matrices $H$ (cf. (1.11) below), we obtain that $\lambda=1$ is the only bifurcation point belonging to our continuum (in other words, the only trivial solution $u \equiv 0$ belonging to the continuum is the one associated with the eigenvalue $\lambda=1$, from which we are bifurcating). For more details we refer to Remark 2.7 below.

We finally impose that $\nu(L)=0$ (cf. condition (2.11)), in order to construct the Green's function associated with $u(t)^{\prime \prime}+L(t) u(t)=0$.

Before presenting our main result, let us associate with each solution of problem (1.1) an index and a nullity. In particular, given a solution $(\lambda, u)$ of problem (1.1), we consider the eigenvalue problem

$$
z^{\prime \prime}(t)+(A(t, u(t), \lambda)+\eta \mathrm{Id}) z(t)=0, \quad z(0)=0=z(T),
$$

and, by focusing our attention on the corresponding index and nullity, we set

$$
i(\lambda, u):=i(A(\cdot, u(\cdot), \lambda)) \quad \text { and } \quad \nu(\lambda, u):=\nu(A(\cdot, u(\cdot), \lambda)) .
$$

Our choice of characterizing the lower and upper bounds on the nonlinearity by two consecutive indexes guarantees the preservation of the index associated with 
each nontrivial solution of problem (1.1). Moreover, our assumptions ensure that the nullity of each nontrivial solution of (1.1) is equal to 1 (roughly speaking, this means that the eigenspace associated with each nontrivial solution has dimension 1) and this is the simplest context where the Rabinowitz global bifurcation theorem can be applied.

Note that preservation results of the Maslov index along global bifurcating branches of solutions have been achieved in the work [2], handling first order systems.

There exists an extensive literature concerning bifurcation results for various classes of perturbed elliptic systems. In this framework, many authors have provided the existence of a continuum of positive solutions bifurcating from the simple, principal eigenvalue of an associated unperturbed system, characterized by being the unique positive eigenvalue having a positive eigenfunction. Among the various reference concerning this topic, we wish to quote [5], where Rabinowitz global bifurcation theorem is applied to find positive solutions to a class of elliptic systems when cooperative and fully coupled assumptions hold. We also refer to the papers [24] and [26] dealing with bifurcation phenomena arising in quasilinear elliptic systems involving $(p, q)$-Laplacian operators.

Bifurcation techniques have been also employed in [15] to prove the existence of an unbounded set of positive solutions emanating from a positive eigenvalue of the unperturbed system having a positive eigenfunction, when simplicity and uniqueness of this eigenvalue are not guaranteed. We wish also to mention the paper [12], devoted to the study of the structure of the connected set of nontrivial periodic solutions of periodic perturbations of second order autonomous ODEs on differentiable manifolds. Interesting contributions can be also found, among others, in the works [10] and [11], where bifurcation methods are used to exhibit the existence of a continuum of positive solutions arising in elliptic systems depending on various parameters.

Let us now introduce one more notation. According to [8], for any $A, B \in$ $M_{s}(n)$, we write $A \leq B$ if $B-A$ is positively semi-definite and $A<B$ if $B-A$ is positive definite. Moreover, for any $A, B \in L^{\infty}\left([0, T] ; M_{s}(n)\right)$ by $A \leq B$ we mean that $A(t) \leq B(t)$ for a.e. $t \in[0, T]$ and by $A<B$ we mean that $A(t) \leq B(t)$ for a.e. $t \in[0, T]$ and $A(t)<B(t)$ on a subset of $[0, T]$ with positive measure.

We are now in position to state our main Theorem.

Theorem 1.3. Consider $A \in C\left([0, T] \times \mathbb{R}^{n} \times \mathbb{R} ; M_{s}(n)\right)$ defined by (1.2) and assume the existence of $r>1$ and of $B \in L^{\infty}\left([0, T] ; M_{s}(n)\right)$ with

$$
i(B)=1
$$

such that

$$
A(\cdot, x, \lambda)<B(\cdot) \quad \forall x \in \mathbb{R}^{n}, \forall \lambda \in(-\infty, r) .
$$


Suppose moreover that

$$
\begin{aligned}
\nu(L+H) & \neq 0, \\
H & >0 .
\end{aligned}
$$

Then, there exists a continuum of solutions $\mathcal{C} \subset(-\infty, r) \times C^{2}\left([0, T] ; \mathbb{R}^{n}\right)$ of problem $(1.1)$ containing $(1,0)$ and such that there exists $\left(\lambda_{m}, u_{m}\right) \in \mathcal{C}$ satisfying

$$
\text { either }\left|\lambda_{m}\right|+\left\|u_{m}\right\| \rightarrow+\infty \text { or } \lambda_{m} \rightarrow r \text {. }
$$

Moreover, $\mathcal{C} \backslash\{(1,0)\}$ is composed by nontrivial solutions of problem (1.1), and

$$
i(\lambda, u)=0, \quad \nu(\lambda, u)=1 \quad \forall(\lambda, u) \in \mathcal{C} .
$$

We observe that an analogous result can be achieved by varying the bounds in (1.9) and their indexes in (1.8). For further details, we refer to Theorem 2.10.

This work is organized as follows. In Sect. 2 we present some preliminary results concerning index and nullity, we prove Theorem 1.3 and we present a variant version of its statement. Section 3 is devoted to analyze the behaviour of the branches of solutions to problem (1.1) when some additional assumptions on the nonlinearity are imposed. In Sect. 4, besides recalling the Lazer's result [17] and providing some remark devoted to clarify the choice of our assumptions, we develop an application of our result to the study of branches of odd, periodic solutions for suitable second order systems, modeling two pendulums coupled by a torsion elastic force, in presence of odd, periodic disturbances of their lengths (cf. Theorems 4.7 and 4.9).

\section{Main results}

In the first part of this section we present some preliminary results and observations, needed to prove Theorem 1.3.

Let us first recall a Sturm comparison result proved by Dong in [8] which will be used throughtout this work

Proposition 2.1. (cf. [8, Proposition 2.6]) For every $A, B \in L^{\infty}\left([0, T] ; M_{s}(n)\right)$, if $A \leq B$, then $i(A) \leq i(B)$; if $A<B$, then $i(A)+\nu(A) \leq i(B)$.

As a first consequence of Proposition 2.1, we can determinate the exact values of the index and nullity of $H$. More precisely, we get

Proposition 2.2. Assume (1.8)-(1.10). Then,

$$
\nu(L+H)=1 \quad \text { and } \quad i(L+H)=0 .
$$

Proof. Let us recall the definition of $A$ given in (1.2) and let us restrict the condition (1.9) to the case $x=0$, taking into account that $R(t, 0, \lambda) \equiv 0$ and $1<r$. We immediately deduce that $L+H<B$. Hence, combining Proposition 2.1 with assumption (1.8) we obtain

$$
\nu(L+H) \leq i(L+H)+\nu(L+H) \leq i(B)=1 .
$$

From hypothesis (1.10), the thesis follows. 
Remark 2.3. By combining Propositions 2.1 and 2.2 with assumption (1.11), we infer that $i(L)=\nu(L)=0$.

With analogous arguments based on the application of Proposition 2.1, we will show that the index and the nullity are preserved along all the possible branches of nontrivial solutions $(\lambda, u)$ of problem (1.1) having $\lambda<r$. According to the notation introduced in (1.7), we can state the following Proposition.

Proposition 2.4. Assume (1.8) and (1.9). Then, every solution $(\lambda, u) \in(-\infty, r) \times$ $C^{2}\left([0, T] ; \mathbb{R}^{n}\right)$ of problem $(1.1)$ with $u \neq \equiv 0$ satisfies

$$
i(\lambda, u)=0 \quad \text { and } \quad \nu(\lambda, u)=1 .
$$

Proof. Consider a solution $(\lambda, u)$ of problem (1.1) with $\lambda<r$ and $u \not \equiv 0$.

Taking into account the definition of $A$ given in (1.2) and the assumption (1.9), it immediately follows that

$$
A(\cdot, u(\cdot), \lambda)<B(\cdot),
$$

which, according to the notation introduced in (1.7), to Proposition 2.1 and to hypothesis (1.8), implies that

$$
\nu(\lambda, u) \leq i(\lambda, u)+\nu(\lambda, u) \leq i(B)=1 .
$$

Moreover, observe that $u$ is a nontrivial solution of the Dirichlet problem

$$
z^{\prime \prime}(t)+A(t, u(t), \lambda) z(t)=0, \quad z(0)=0=z(T),
$$

and, consequently, $\eta=0$ is an eigenvalue of problem (1.6). By the definition of nullity, we can finally conclude that

$$
1 \leq \nu(\lambda, u),
$$

which, combined with (2.3), leads to (2.2). The thesis is achieved.

Finally, we present two well-known results on which the proof of our main theorem is based. The first proposition is concerned with the continuity of the $j$ th eigenvalue of problem (1.3).

Proposition 2.5. (cf. [6, Corollary 2.5]) Fixed $M>0$, for each $j=1, \ldots,+\infty$, the map $K \rightarrow \eta_{j}(K)$ is continuous in $\left\{K \in L^{1}\left([0, T] ; M_{s}(n)\right):\|K(t)\|<\right.$ $M$ for a.e. $t \in(0, T)\}$.

The proof of this proposition, exhibited in [6], is based on some arguments developed by de Figueiredo in the paper [7], describing eigenvalues and eigenfunctions of linear elliptic problems with indefinite weights in a PDEs setting. The proof in [6] deals with planar systems, but it admits an easy extension to higher dimensions.

Another crucial tool is the classical Rabinowitz bifurcation theorem. Let us recall its statement. 
Theorem 2.6. ( $c f$. [23]). Let $E$ be a Banach space with norm $\|\cdot\|$. Consider $r_{1}, r_{2} \in$ $\overline{\mathbb{R}}$ with $r_{1}<r_{2}$, a compact linear map $\mathcal{L}$ on $E$ and a compact function $\mathcal{R}$ : $\left(r_{1}, r_{2}\right) \times E \rightarrow E$ satisfying $\mathcal{R}(\lambda, x)=o(\|x\|)$ as $x \rightarrow 0$, uniformly on bounded $\lambda$ intervals. Denote by $\mathcal{S}$ the closure of the set of nontrivial solutions of the equation

$$
u=\lambda \mathcal{L} u+\mathcal{R}(\lambda, u) .
$$

If $\mu_{0} \in\left(r_{1}, r_{2}\right)$ is a characteristic value of $\mathcal{L}$ of odd multiplicity, then $\mathcal{S}$ possesses a maximal subcontinuum $\mathcal{C}$ containing $\left(\mu_{0}, 0\right)$ and such that either

(i) there exists $\left(\lambda_{n}, u_{n}\right) \in \mathcal{C}$ satisfying

$$
\left|\lambda_{n}\right|+\left\|u_{n}\right\| \rightarrow+\infty \quad \text { or } \quad \lambda_{n} \rightarrow r_{1} \quad \text { or } \quad \lambda_{n} \rightarrow r_{2}
$$

or

(ii) there exists a characteristic value $\hat{\mu} \in\left(r_{1}, r_{2}\right)$ with $\hat{\mu} \neq \mu_{0}$ and $(\hat{\mu}, 0) \in \mathcal{C}$.

We are now in position to prove our main Theorem.

Proof of Theorem 1.3. As a first step, we prove the applicability of Theorem 2.6 with $\mu_{0}=1, r_{1}=-\infty, r_{2}=r$, under the assumptions (1.8)-(1.11).

To this aim, we are interested in rewriting problem (1.1) in the form (2.4). Let us concentrate on the linear system

$$
u^{\prime \prime}(t)+L(t) u(t)=0 .
$$

For every $i \in\{1, \ldots, n\}$, let us denote by $u^{i}$ and $v^{i}$ the solutions of (2.5) satisfying

$$
u^{i}(0)=0=v^{i}(T)
$$

and

$$
\left[\left(u^{1}\right)^{\prime}(0) \ldots\left(u^{n}\right)^{\prime}(0)\right]=\operatorname{Id}=\left[\left(v^{1}\right)^{\prime}(T) \ldots\left(v^{n}\right)^{\prime}(T)\right]
$$

If we set

$$
\widetilde{u}^{i}=\left[\begin{array}{c}
u^{i} \\
\left(u^{i}\right)^{\prime}
\end{array}\right], \quad \widetilde{v}^{i}=\left[\begin{array}{c}
v^{i} \\
\left(v^{i}\right)^{\prime}
\end{array}\right] \quad \forall i \in\{1, \ldots, n\},
$$

it is immediate to note that $\widetilde{u}^{1}, \ldots, \widetilde{u}^{n}$ are linearly independent, as well as $\widetilde{v}^{1}, \ldots, \widetilde{v}^{n}$.

Our next purpose consists in showing that all these $2 n$ vectors are linearly independent. Assume that there exist $2 n$ real constants $\alpha_{1}, \ldots, \alpha_{n}, \beta_{1}, \ldots, \beta_{n}$ such that

$$
\sum_{i=1}^{n} \alpha_{i} \widetilde{u}^{i}(t)+\sum_{i=1}^{n} \beta_{i} \widetilde{v}^{i}(t)=0 \quad \forall t \in[0, T] .
$$

Let us define

$$
z(t):=\sum_{i=1}^{n} \alpha_{i} u^{i}(t) \quad \text { and } \quad \eta(t)=\sum_{i=1}^{n} \beta_{i} v^{i}(t) .
$$


Since $z(0)=\eta(T)=0$, from $(2.6)$ it follows that $z(T)=\eta(0)=0$. Thus, $z$ and $\eta$ are both solutions of the problem

$$
u^{\prime \prime}(t)+L(t) u(t)=0, \quad u(0)=0=u(T) .
$$

Since $\nu(L)=0$ by Remark 2.3, we deduce that $z \equiv \eta \equiv 0$, which, according to the definitions of $\widetilde{u}^{i}$ and $\widetilde{v}^{i}$, leads to

$$
\sum_{i=1}^{n} \alpha_{i} \widetilde{u}^{i}(t)=0 \quad \text { and } \quad \sum_{i=1}^{n} \beta_{i} \widetilde{v}^{i}(t)=0 \quad \forall t \in[0, T] .
$$

Due to the linear independence of $\widetilde{u}^{i}, \ldots, \widetilde{u}^{n}$ and $\widetilde{v}^{i}, \ldots, \widetilde{v}^{n}$ separately, we finally conclude that

$$
\alpha_{i}=\beta_{i}=0 \quad \forall i \in\{1, \ldots, n\} .
$$

The linear independence of $\widetilde{u}^{1}, \ldots, \widetilde{u}^{n}, \widetilde{v}^{1}, \ldots, \widetilde{v}^{n}$ follows.

In particular, the $2 n \times 2 n$ matrix defined by

$$
Y(t):=\left[\widetilde{u}^{1}(t) \cdots \widetilde{u}^{n}(t) \widetilde{v}^{1}(t) \cdots \widetilde{v}^{n}(t)\right]
$$

is nonsingular. Moreover, $Y(t)$ and its inverse $Y^{-1}(t)$ can be represented through blocks of $n \times n$ matrices as follows

$$
Y(t):=\left[\begin{array}{ll}
U(t) & V(t) \\
U^{\prime}(t) & V^{\prime}(t)
\end{array}\right] \quad Y^{-1}(t):=\left[\begin{array}{ll}
W_{1}(t) & W_{2}(t) \\
W_{3}(t) & W_{4}(t)
\end{array}\right] .
$$

Taking into account [14] and [16], it is easy to determinate the Green's function $K(s, t)$ associated with the problem (2.5) under the Dirichlet boundary conditions $u(0)=0=u(T)$. More precisely, it is explicitly given by the following $n \times n$ matrix

$$
K(t, s)= \begin{cases}V(t) W_{4}(s) & \text { if } 0 \leq s \leq t \leq T \\ -U(t) W_{2}(s) & \text { if } 0 \leq t \leq s \leq T\end{cases}
$$

Note that, by construction, $K$ is well defined and continuous on $[0, T] \times[0, T]$. We can set $E:=C\left([0, T] ; \mathbb{R}^{n}\right)$,

$$
\begin{aligned}
\mathcal{L}(u)(t) & :=-\int_{0}^{T} K(t, s) H(s) u(s) d s \\
\mathcal{R}(\lambda, u)(t) & :=-\int_{0}^{T} K(t, s) R(s, u(s), \lambda) u(s) d s,
\end{aligned}
$$

for any $t \in[0, T], u \in E, \lambda \in(-\infty, r)$, where $H$ and $R$ have been introduced in (1.2). It is easy to check that $\mathcal{L}: E \rightarrow C^{2}\left([0, T] ; \mathbb{R}^{n}\right) \subset E$ is a compact linear map. Moreover, taking into account that $R(t, x, \lambda) x=o(\|x\|)$ as $x \rightarrow 0$, uniformly on $t \in[0, T]$ and on bounded $\lambda$ intervals, we immediately deduce that $\mathcal{R}$ : $(-\infty, r) \times E \rightarrow C^{2}\left([0, T] ; \mathbb{R}^{n}\right) \subset E$ is a compact map satisfying $\mathcal{R}(\lambda, x)=o(\|x\|)$ as $x \rightarrow 0$, uniformly on bounded $\lambda$ intervals. Finally, observe that problem (1.1) can be rewritten in the form (2.4).

Proposition 2.2 guarantees that $\nu(L+H)=1$. This means that the space of solutions of problem $u^{\prime \prime}+L(t) u+H(t) u=0, u(0)=0=u(T)$ has dimension 1 . As a consequence, 1 is a characteristic value of $\mathcal{L}$ and it is simple. 
All the assumptions of Theorem 2.6 are satisfied. Hence, Theorem 2.6 ensures the existence of a continuum of solutions $\mathcal{C} \subset(-\infty, r) \times C^{2}\left([0, T] ; \mathbb{R}^{n}\right)$ of problem (1.1) containing $(1,0)$ and satisfying either (i) or (ii).

The validity of $(1.13)$ when $(\lambda, u)=(1,0)$ immediately follows by an application of Proposition 2.2 combined with the fact that $A(\cdot, 0,1)=L+H$.

Furthermore, Proposition 2.4 ensures that $i(\lambda, u)=0$ and $\nu(\lambda, u)=1$ for every $(\lambda, u) \in \mathcal{C}$ with $u \neq \equiv$. As a consequence, the eigenvalue problem (1.6) does not admit any negative eigenvalue and its first eigenvalue is zero. Equivalently,

$$
\eta_{1}(A(\cdot, u(\cdot), \lambda))=0 \quad \forall(\lambda, u) \in \mathcal{C}: u \neq \equiv .
$$

Taking into account the continuity of the first eigenvalue established by Proposition 2.5 and recalling that $\mathcal{C}$ is a continuum, we infer that

$$
\eta_{1}(A(\cdot, u(\cdot), \lambda))=0 \quad \forall(\lambda, u) \in \mathcal{C},
$$

which, according to Definition 1.2, leads to

$$
i(A(\cdot, u(\cdot), \lambda))=0 \quad \text { and } \quad \nu(A(\cdot, u(\cdot), \lambda)) \geq 1 \quad \forall(\lambda, u) \in \mathcal{C} .
$$

To complete the proof of Theorem 1.3, we need to prove that in our case the alternative (ii) cannot occur. Assume by contradiction that alternative (ii) is satisfied, and, consequently, there exists $\hat{\mu} \in(-\infty, r)$ with $\hat{\mu} \neq 1$ satisfying $(\hat{\mu}, 0) \in \mathcal{C}$. Since $A(\cdot, 0, \hat{\mu})=L+\hat{\mu} H$, from (2.1) and (2.9) it immediately follows that

$$
i(L+H)=0, \quad \nu(L+H)=1, \quad i(L+\hat{\mu} H)=0, \quad \nu(L+\hat{\mu} H) \geq 1 .
$$

Since $H>0$ by assumption (1.11), we observe that for every $\mu_{1}, \mu_{2} \in \mathbb{R}$

$$
\mu_{1}<\mu_{2} \Longrightarrow \mu_{1} H<\mu_{2} H \text {. }
$$

As a consequence, Proposition 2.1 ensures that

$$
i\left(L+\mu_{1} H\right)+\nu\left(L+\mu_{1} H\right) \leq i\left(L+\mu_{2} H\right) \text { if } \mu_{1}<\mu_{2},
$$

which contradicts (2.10). This excludes alternative (ii) and completes the proof of Theorem 1.3.

Remark 2.7. We point out that we do not need to use hypothesis (1.11) to guarantee the existence of a continuum of solutions $\mathcal{C} \subset(-\infty, r) \times C^{2}\left([0, T] ; \mathbb{R}^{n}\right)$ of problem (1.1) containing $(1,0)$ and satisfying either $(i)$ or $(i i)$, provided that we assume

$$
\nu(L)=0 .
$$

We employ assumption (1.11) just to prove that alternative (ii) cannot occur.

Remark 2.8. It is possible to reformulate the statement of Theorem 1.3 in order to find a continuum of solutions bifurcating from $\left(\lambda_{0}, 0\right)$, provided that we take $r>\lambda_{0}$ and that we replace assumption (1.10) with the condition $\nu\left(L+\lambda_{0} H\right) \neq 0$. If $\lambda_{0}<0$, we have to assume the additional hypothesis $\nu(L)=0$. 
Remark 2.9. An example of path of symmetric matrices verifying the condition (1.8) is given by the constant path

$$
B(t)=B=\operatorname{diag}\left(w_{1}, \ldots, w_{n}\right),
$$

where $w_{1} \in\left(\frac{\pi^{2}}{T^{2}}, \frac{4 \pi^{2}}{T^{2}}\right]$ and $w_{2}, \ldots, w_{n} \in\left(-\infty, \frac{\pi^{2}}{T^{2}}\right]$.

The assumptions (1.8)-(1.9) of Theorem 1.3 play a crucial role to determine branches of solutions of problem (1.1) whose index is equal to zero. We are now interested in extending Theorem 1.3 in order to obtain branches of solutions with non zero index. To this aim, assume that $\nu(L)=0$ and that $A(\cdot, x, \lambda)$ is bounded by two paths of symmetric matrices whose indexes are two consecutive natural numbers. Arguing as in the proof of Theorem 1.3, we can show that the index of the solutions to problem (1.1) coincides with the index associated with the lower bound. More precisely, the following result holds.

Theorem 2.10. Consider $A \in C\left([0, T] \times \mathbb{R}^{n} \times \mathbb{R} ; M_{s}(n)\right)$ defined by (1.2) satisfying (1.10), (1.11) and (2.11). Assume the existence of $k \in \mathbb{N} \cup\{0\}, r_{1}, r_{2} \in \overline{\mathbb{R}}$ with $r_{1}<1<r_{2}, B_{1}, B_{2} \in L^{\infty}\left([0, T] ; M_{s}(n)\right)$ with

$$
i\left(B_{1}\right)=k, \quad i\left(B_{2}\right)=k+1
$$

such that

$$
B_{1}(\cdot)<A(\cdot, x, \lambda)<B_{2}(\cdot) \quad \forall x \in \mathbb{R}^{n}, \quad \forall \lambda \in\left(r_{1}, r_{2}\right) .
$$

Then, there exists a continuum of solutions $\mathcal{C} \subset\left(r_{1}, r_{2}\right) \times C^{2}\left([0, T] ; \mathbb{R}^{n}\right)$ of problem (1.1) containing $(1,0)$ and such that there exists $\left(\lambda_{m}, u_{m}\right) \in \mathcal{C}$ satisfying

$$
\text { either }\left|\lambda_{m}\right|+\left\|u_{m}\right\| \rightarrow+\infty \quad \text { or } \quad \lambda_{m} \rightarrow r_{j} \text { with } j \in\{1,2\} \text {. }
$$

Moreover, $\mathcal{C} \backslash\{(1,0)\}$ is composed by nontrivial solutions of problem (1.1), and

$$
i(\lambda, u)=k, \quad \nu(\lambda, u)=1 \quad \forall(\lambda, u) \in \mathcal{C} .
$$

Let us recall that an analogous of Proposition 2.2 can be stated under the assumptions (2.12) and (2.13). More precisely, the following proposition is satisfied

Proposition 2.11. Assume (1.10), (2.12)-(2.13). Then,

$$
\nu(L+H)=1 \quad \text { and } \quad i(L+H)=k .
$$

Remark 2.12. According to Proposition 2.2 and Proposition 2.11, we can equivalently state Theorem 1.3 and Theorem 2.10 by replacing assumption (1.10) with the assumption

$$
\nu(L+H)=1 .
$$




\section{Refinements of the main results}

This section is devoted to present various kind of additional assumptions which could be imposed on the nonlinearity to refine the alternatives (1.12) and (2.14) in the theses of our main theorems.

Before stating our corollaries, we need to introduce a notation. Given a continuum $\mathcal{C} \subset\left(r_{1}, r_{2}\right) \times C^{2}\left([0, T] ; \mathbb{R}^{n}\right)$, let us denote by $\Pi_{1}(\mathcal{C})$ its projection on the open interval $\left(r_{1}, r_{2}\right)$ and by $\Pi_{2}(\mathcal{C})$ its projection on the set $C^{2}\left([0, T] ; \mathbb{R}^{n}\right)$. We first analyze the case in which the path of symmetric matrices $R$ in (1.2) is required to be bounded from above. This assumption guarantees the existence of a continuum $\mathcal{C}$ of solutions of problem $(1.1)$ in which $\Pi_{1}(\mathcal{C})$ is bounded whenever $\Pi_{2}(\mathcal{C})$ is bounded.

Corollary 3.1. Besides the assumptions of Theorem 1.3, suppose that there exists a continuous map $R_{0}:[0, T] \times \mathbb{R}^{n} \rightarrow M_{s}(n)$ such that

$$
R(\cdot, x, \lambda) \leq R_{0}(\cdot, x) \quad \forall x \in \mathbb{R}^{n}, \lambda \in(-\infty, r)
$$

Then, there exists a continuum of solutions $\mathcal{C} \subset(-\infty, r) \times C^{2}\left([0, T] ; \mathbb{R}^{n}\right)$ of problem (1.1) containing $(1,0)$, satisfying $(1.13)$, and such that $\Pi_{1}(\mathcal{C})$ is bounded whenever $\Pi_{2}(\mathcal{C})$ is bounded. Moreover, there exists $\left(\lambda_{m}, u_{m}\right) \in \mathcal{C}$ satisfying

$$
\text { either }\left\|u_{m}\right\| \rightarrow+\infty \text { or } \lambda_{m} \rightarrow r \text {. }
$$

Proof. Theorem 1.3 guarantees the existence of a continuum of solutions $\mathcal{C} \subset$ $(-\infty, r) \times C^{2}\left([0, T] ; \mathbb{R}^{n}\right)$ of problem (1.1) containing $(1,0)$ and satisfying (1.13).

Let $\Pi_{2}(\mathcal{C})$ be bounded, i.e. let exist $c>0$ such that $\|u\| \leq c$ for every $u \in \Pi_{2}(\mathcal{C})$. Our aim consists in showing that, under this assumption, $\Pi_{1}(\mathcal{C})$ is bounded too. Assume, by contradiction, that there exists a sequence $\lambda_{m} \in \Pi_{1}(\mathcal{C})$ such that $\lambda_{m} \rightarrow-\infty$ as $m \rightarrow+\infty$. In particular, there exists $u_{m} \in C^{2}\left([0, T] ; \mathbb{R}^{n}\right)$ such that $\left(\lambda_{m}, u_{m}\right) \in \mathcal{C}$. By (1.2) and (3.1), it follows that for every $x \in \mathbb{R}^{n}$, $m \in \mathbb{N}$

$$
A\left(\cdot, x, \lambda_{m}\right)=L(\cdot)+\lambda_{m} H(\cdot)+R\left(\cdot, x, \lambda_{m}\right) \leq L(\cdot)+\lambda_{m} H(\cdot)+R_{0}(\cdot, x) .
$$

According to the continuity of $R_{0}$, the assumption (1.11) and the fact that $\lambda_{m} \rightarrow$ $-\infty$ as $m \rightarrow+\infty$, we infer the existence of $m_{0}=m_{0}(c) \in \mathbb{N}$ such that for every $m \geq m_{0}$ we have

$$
L(t)+\lambda_{m} H(t)+R_{0}(t, x)<0 \quad \forall m \geq m_{0}, t \in[0, T], x \in \mathbb{R}^{n}:\|x\| \leq c .
$$

Hence, by taking into account (3.3) and Proposition 2.1, we conclude that

$$
i\left(\lambda_{m}, u_{m}\right)+\nu\left(\lambda_{m}, u_{m}\right) \leq i(0)=0 \quad \forall m \geq m_{0}
$$

which contradicts $(1.13)$. We have so proved that the boundedness of $\Pi_{2}(\mathcal{C})$ implies the boundedness of $\Pi_{1}(\mathcal{C})$.

In order to complete the proof, it remains to prove that there exists $\left(\lambda_{m}, u_{m}\right) \in \mathcal{C}$ satisfying (3.2). Theorem 1.3 ensures the existence of $\left(\lambda_{m}, u_{m}\right) \in \mathcal{C}$ satisfying either $\left|\lambda_{m}\right|+\left\|u_{m}\right\| \rightarrow+\infty$ or $\lambda_{m} \rightarrow r$. If the first alternative holds, 
then, by the previous step, the sequence $u_{m}$ cannot be bounded and therefore admits a subsequence $u_{k_{m}}$ with $\left\|u_{k_{m}}\right\| \rightarrow+\infty$ as $m \rightarrow+\infty$.

Remark 3.2. By repeating the same arguments developed in the previous proof, we can show that Corollary 3.1 holds true if we replace assumption (3.1) with the more general assumption

$$
\forall c>0 \quad \exists M_{c}>0: A(\cdot, x, \lambda)<0 \quad \forall x \in \mathbb{R}^{n}:\|x\| \leq c, \lambda \in\left(-\infty,-M_{c}\right] .
$$

The following corollary establishes the boundedness of $\Pi_{1}(\mathcal{C})$ when suitable upper estimates on the path of symmetric matrices $A$ are satisfied.

Corollary 3.3. Besides the assumptions of Theorem 1.3, suppose that there exist $\lambda_{0}<1$ and $K \in L^{\infty}\left([0, T] ; M_{s}(n)\right)$ such that

$$
i(K)=0 \quad \text { and } \quad A\left(\cdot, x, \lambda_{0}\right)<K(\cdot) \quad \forall x \in \mathbb{R}^{n} .
$$

Then, there exists a continuum of solutions $\mathcal{C} \subset\left(\lambda_{0}, r\right) \times C^{2}\left([0, T] ; \mathbb{R}^{n}\right)$ of problem (1.1) containing $(1,0)$ and satisfying $(1.13)$. Moreover, there exists $\left(\lambda_{m}, u_{m}\right) \in \mathcal{C}$ satisfying (3.2).

Proof. By Theorem 1.3, there exists a continuum of solutions $\mathcal{C} \subset(-\infty, r) \times$ $C^{2}\left([0, T] ; \mathbb{R}^{n}\right)$ of problem $(1.1)$ containing $(1,0)$ and satisfying (1.13). To complete the proof, we need to verify that $\Pi_{1}(\mathcal{C}) \subset\left(\lambda_{0}, r\right)$. Let us argue by contradiction, assuming that there exists $\tilde{\lambda} \leq \lambda_{0}$ with $\tilde{\lambda} \in \Pi_{1}(\mathcal{C})$. Being $\mathcal{C}$ a closed connected set in $(-\infty, r) \times C^{2}\left([0, T] ; \mathbb{R}^{n}\right)$, its projection $\Pi_{1}(\mathcal{C})$ is a connected set in $(-\infty, r)$ and, consequently, it is an interval. In particular, $\lambda_{0} \in[\tilde{\lambda}, 1) \subset[\tilde{\lambda}, 1] \subset \Pi_{1}(\mathcal{C})$. Let $u_{0} \in C^{2}\left([0, T] ; \mathbb{R}^{n}\right)$ be such that $\left(\lambda_{0}, u_{0}\right) \in \mathcal{C}$. By combining assumption $(3.4)$ with Proposition 2.1, we infer that

$$
i\left(\lambda_{0}, u_{0}\right)+\nu\left(\lambda_{0}, u_{0}\right) \leq i(K)=0 .
$$

Thus, $\nu\left(\lambda_{0}, u_{0}\right)=0$, which contradicts (1.13). The proof is complete.

Remark 3.4. Condition (3.4) holds if we assume the validity of (1.11) and suppose the existence of a continuous map $R_{0}:[0, T] \rightarrow M_{s}(n)$ such that

$$
R(\cdot, x, \lambda) \leq R_{0}(\cdot) \quad \forall x \in \mathbb{R}^{n}, \lambda \in(-\infty, r) .
$$

Indeed, according to the assumptions (1.11) and (3.5), it is immediate to check that there exists a suitable negative constant $\lambda_{0}$ such that

$$
A(\cdot, x, \lambda)=L(\cdot)+\lambda H(\cdot)+R(\cdot, x, \lambda) \leq L+\lambda H+R_{0}<0 \quad \forall x \in \mathbb{R}^{n}, \lambda \leq \lambda_{0} .
$$

Let us now present some additional hypotheses providing further refinements of the alternatives (1.12) and (2.14) in the thesis of our main theorems. In particular, the following corollaries establish the existence of bounded branches of solutions to problem (1.1). 
Corollary 3.5. Besides the assumptions of Theorem 2.10 where we take $r_{1}, r_{2} \in \mathbb{R}$, suppose the existence of $A_{\infty} \in C\left([0, T] ; M_{s}(n)\right)$ such that

$$
\begin{gathered}
\nu\left(A_{\infty}\right)=0 \\
\lim _{\|x\| \rightarrow \infty} A(t, x, \lambda)=A_{\infty}(t) \quad \text { uniformly in }(t, \lambda) \in[0, T] \times\left[r_{1}, r_{2}\right] .
\end{gathered}
$$

Then, there exists a bounded continuum of solutions $\mathcal{C} \subset\left(r_{1}, r_{2}\right) \times C^{2}\left([0, T] ; \mathbb{R}^{n}\right)$ of problem (1.1) containing $(1,0)$, such that $(2.15)$ holds, and there exists $\left(\lambda_{m}, u_{m}\right) \in$ $\mathcal{C}$ satisfying

$$
\text { either } \lambda_{m} \rightarrow r_{1} \quad \text { or } \quad \lambda_{m} \rightarrow r_{2} \text {. }
$$

Proof. Theorem 2.10 guarantees the existence of a continuum of solutions $\mathcal{C} \subset$ $\left(r_{1}, r_{2}\right) \times C^{2}\left([0, T] ; \mathbb{R}^{n}\right)$ of problem (1.1) satisfying (2.15). Assume by contradiction that there exists a sequence $\left(\lambda_{m}, u_{m}\right) \in \mathcal{C}$ with $\left\|u_{m}\right\| \rightarrow+\infty$ as $m \rightarrow+\infty$. Condition (2.15) ensures that

$$
\eta_{k+1}\left(A\left(\cdot, u_{m}(\cdot), \lambda_{m}\right)\right)=0 .
$$

Moreover, assumption (3.7) combined with the continuity of $A$ implies that there exists $M>0$ such that

$$
\|A(t, x, \lambda)\|<M \quad \forall t \in[0, T], \forall x \in \mathbb{R}^{n}, \forall \lambda \in\left[r_{1}, r_{2}\right] .
$$

Condition (3.9) guarantees the applicability of the "Elastic Lemma" (cf., for instance, Lemma 2.1 in [13]), which asserts that for every $R_{1}>0$ there exists $R_{2}>$ $R_{1}$ such that for every solution $(\lambda, u)$ of $(1.1)$ with $\min _{t \in[0, T]}\left\|\left(u(t), u^{\prime}(t)\right)\right\| \leq R_{1}$ it follows that $\left\|\left(u(t), u^{\prime}(t)\right)\right\| \leq R_{2}$ for every $t \in[0, T]$. As a consequence, from the fact that $\left\|u_{m}\right\| \rightarrow+\infty$ as $m \rightarrow+\infty$, we deduce that, up to a subsequence, $\left\|u_{m}^{\prime}(0)\right\| \rightarrow+\infty$ as $m \rightarrow+\infty$. Taking into account (3.9), we finally can apply Proposition 4.4 in [3] to deduce that

$$
A\left(t, u_{m}(t), \lambda_{m}\right) \rightarrow A_{\infty}(t) \quad \text { in } L^{1}\left([0, T] ; M_{s}(n)\right) \quad \text { as } m \rightarrow+\infty .
$$

Thus, by combining Proposition 2.5 with (3.8)-(3.10), we infer that

$$
\eta_{k+1}\left(A_{\infty}\right)=\lim _{m \rightarrow+\infty} \eta_{k+1}\left(A\left(\cdot, u_{m}(\cdot), \lambda_{m}\right)\right)=0
$$

which contradicts (3.6). The thesis follows.

Arguing as above and taking into account Corollary 3.3 and Remark 3.4, we can prove the following result.

Corollary 3.6. Besides the assumptions of Theorem 1.3, suppose the validity of (3.5). Furthermore, assume the existence of $A_{\infty} \in C\left([0, T] ; M_{s}(n)\right)$ satisfying $(3.6)$ and

$$
\lim _{\|x\| \rightarrow \infty} A(t, x, \lambda)=A_{\infty}(t) \quad \text { uniformly in }(t, \lambda) \in[0, T] \times(-\infty, r] .
$$


Then, there exists a bounded continuum of solutions $\mathcal{C} \subset(-\infty, r) \times C^{2}\left([0, T] ; \mathbb{R}^{n}\right)$ of problem (1.1) containing (1,0), such that (1.13) holds and there exists $\left(\lambda_{m}, u_{m}\right) \in \mathcal{C}$ satisfying

$$
\lambda_{m} \rightarrow r .
$$

We are now interested in exhibiting a particular situation in which assumption (3.4) is satisfied. To this aim, let us consider the parameter-dependent problem

$$
\left\{\begin{array}{l}
u^{\prime \prime}(t)+L(t) u(t)+\lambda \nabla_{u} G(t, u(t))=0 \\
u(0)=0=u(T)
\end{array}\right.
$$

where $L \in C\left([0, T] ; M_{s}(n)\right), G \in C^{2}\left([0, T] \times \mathbb{R}^{n} ; \mathbb{R}\right)$ and satisfies

$$
\nabla_{u} G(t, 0)=0 \quad \forall t \in[0, T] .
$$

Denoting by $H$ the Hessian matrix of $G$, and defining $H(t):=H(t, 0)$ for every $t \in[0, T]$, we can state the following result.

Corollary 3.7. Under the assumptions (1.10) and (3.12), suppose the existence of $r>1$ and of $B \in L^{\infty}\left([0, T] ; M_{s}(n)\right)$ satisfying (1.8) and such that

$$
0<r H(\cdot, x) \leq B-L \quad \forall x \in \mathbb{R}^{n} .
$$

Then, there exists a continuum of solutions $\mathcal{C} \subset[0, r) \times C^{2}\left([0, T] ; \mathbb{R}^{n}\right)$ of problem (3.11) containing $(1,0)$, such that $(1.13)$ holds and there exists $\left(\lambda_{m}, u_{m}\right) \in \mathcal{C}$ satisfying (3.2).

Proof. As observed in [3], the system in (3.11) can be written in the form

$$
u^{\prime \prime}(t)+L(t) u(t)+\lambda \mathcal{A}(t, u(t)) u(t)=0,
$$

where

$$
\mathcal{A}(t, x)=\int_{0}^{1} H(t, s x) d s \in M_{s}(n), \quad \forall t \in[0, T], \forall x \in \mathbb{R}^{n} .
$$

From (3.13) we immediately deduce that

$$
0<r \mathcal{A}(\cdot, x) \leq B(\cdot)-L(\cdot) \quad \forall x \in \mathbb{R}^{n},
$$

and, consequently, being $r$ a positive constant,

$$
\mathcal{A}(\cdot, x)>0 \quad \forall x \in \mathbb{R}^{n} .
$$

We are first interested in proving that the nonlinear term is of the form (1.2) and satisfies (1.9), (1.11). According to (3.12) and to the Taylor's formula, we observe that

$$
\nabla_{u} G(t, x)=H(t) x+\widehat{R}(t, x)
$$

with $\widehat{R}(t, x)=o(\|x\|)$ when $x \rightarrow 0, x \in \mathbb{R}^{n}$, uniformly on $t \in[0, T]$. Taking into account (3.14), we can infer that $\widehat{R}(t, x)=(\mathcal{A}(t, x)-H(t)) x$. In particular, if we define

$$
R(t, x, \lambda):=\lambda(\mathcal{A}(t, x)-H(t)) \in M_{s}(n),
$$


we can rewrite problem (3.11) in the equivalent form

$$
\left\{\begin{array}{l}
u^{\prime \prime}(t)+(L(t)+\lambda H(t)+R(t, u(t), \lambda)) u(t)=0 \\
u(0)=0=u(T) .
\end{array}\right.
$$

Notice that $R(t, x, \lambda) x=\lambda \widehat{R}(t, x)=o(\|x\|)$ when $x \rightarrow 0, x \in \mathbb{R}^{n}$, uniformly on $t \in[0, T]$ and on bounded $\lambda$ intervals. Moreover, by (3.17) and (3.16) we can infer that for every $x \in \mathbb{R}^{n}$ and for every $\lambda \in(-\infty, r)$

$$
L(\cdot)+\lambda H(\cdot)+R(\cdot, x, \lambda)=L(\cdot)+\lambda \mathcal{A}(\cdot, x)<L(\cdot)+r \mathcal{A}(\cdot, x) \leq B(\cdot),
$$

which coincides with condition (1.9), provided that we set

$$
A(t, x, \lambda):=L(t)+\lambda H(t)+R(t, x, \lambda)=L(t)+\lambda \mathcal{A}(t, x) .
$$

Recalling that $H(t)=H(t, 0)$, from assumption (3.13) combined with the positiveness of $r$, we deduce the validity of (1.11). Thus, all the assumptions of Theorem 1.3 are satisfied. Moreover, from (3.17), we can assert that

$$
A\left(\cdot, x, \lambda_{0}\right):=L(\cdot)+\lambda_{0} \mathcal{A}(\cdot, x)<L(\cdot) \quad \forall \lambda_{0}<0, x \in \mathbb{R}^{n} .
$$

Since $i(L)=0$ by Remark 2.3, we can apply Corollary 3.3 to achieve the thesis.

Corollary 3.8. Assume (1.10), (1.11), (2.11) and (3.12). Suppose moreover that there exist $k \in \mathbb{N} \cup\{0\}, r_{1}, r_{2} \in \overline{\mathbb{R}}$ with $r_{1}<1<r_{2}, B_{1}, B_{2} \in L^{\infty}\left([0, T] ; M_{s}(n)\right)$ with

$$
i\left(B_{1}\right)=k, \quad i\left(B_{2}\right)=k+1
$$

such that

$$
B_{1}-L<\lambda H(\cdot, x)<B_{2}-L \quad \forall x \in \mathbb{R}^{n}, \forall \lambda \in\left(r_{1}, r_{2}\right) .
$$

Then, there exists a continuum of solutions $\mathcal{C} \subset\left(r_{1}, r_{2}\right) \times C^{2}\left([0, T] ; \mathbb{R}^{n}\right)$ of problem (3.11) containing $(1,0)$, such that $(2.15)$ holds and there exists $\left(\lambda_{m}, u_{m}\right) \in \mathcal{C}$ satisfying (2.14).

Proof. Exactly as in the previous proof, according to (3.12), we can write system (3.11) in the equivalent form (1.1), where $A$ and $R$ are respectively given by (3.19) and (3.18). Recalling the definition of $\mathcal{A}$ introduced in (3.15) and taking into account assumption (3.20), we infer that $B_{1}-L<\lambda \mathcal{A}(\cdot, x)<B_{2}-L$ for every $x \in \mathbb{R}^{n}$ and $\lambda \in\left(r_{1}, r_{2}\right)$. As a consequence,

$$
B_{1}(\cdot)<A(\cdot, x, \lambda)<B_{2}(\cdot) \quad \forall x \in \mathbb{R}^{n}, \forall \lambda \in\left(r_{1}, r_{2}\right) .
$$

The thesis follows by a simple application of Theorem 2.10. 


\section{Some more remarks and an example}

The first part of this section is devoted to clarify our result through some remarks. As mentioned in the introduction, our idea of estimating the nonlinearity by paths of matrices having different indexes (cf. assumptions (1.8) and (1.9) and, particularly, (2.12) and (2.13)) originated from the work [17], where a no-existence result is achieved when the same index is associated with suitable lower and upper bounds. More precisely, by combining a suitable condition for non-degeneracy of symmetric bilinear forms with some properties of Fourier series, Lazer has proved the following theorem

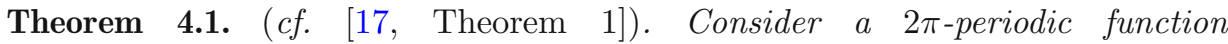
$K \in C\left(\mathbb{R} ; M_{s}(n)\right)$. Assume that there exist $N_{i} \in \mathbb{N}$ with $i \in\{1, \ldots, n\}$ and two constant real symmetric matrices $B_{1}$ and $B_{2}$ with respective eigenvalue $\lambda_{1} \leq \lambda_{2} \leq$ $\cdots \leq \lambda_{n}$ and $\mu_{1} \leq \mu_{2} \leq \cdots \leq \mu_{n}$, satisfying

$$
B_{1}<K(\cdot)<B_{2}
$$

and

$$
N_{i}^{2}<\lambda_{i} \leq \mu_{i}<\left(N_{i}+1\right)^{2} .
$$

Then, there exist no nontrivial $2 \pi$-periodic solutions to

$$
u^{\prime \prime}(t)+K(t) u(t)=0 .
$$

We recall that Ahmad in [1] weakened the continuity assumptions on $K$ by requiring the elements of $K$ to be bounded and measurable. From a direct application of Theorem 4.1, Lazer also deduced the following uniqueness theorem

Theorem 4.2. (cf. [17, Theorem 2]) Consider $G \in C^{2}\left(\mathbb{R}^{n} ; \mathbb{R}\right)$, a $2 \pi$-periodic function $p \in C\left(\mathbb{R} ; \mathbb{R}^{n}\right)$, and denote by $H$ the Hessian matrix of $G$. If there exists two constant symmetric matrices $B_{1}$ and $B_{2}$ satisfying the assumption (4.1) of Theorem 4.1 such that

$$
B_{1}<H(x)<B_{2} \quad \forall x \in \mathbb{R}^{n},
$$

then, there exists at most one $2 \pi$-periodic solution of

$$
u^{\prime \prime}(t)+\nabla G(u)=p(t) .
$$

In particular, if in addition $\nabla G(0)=0$, then there exist no nontrivial $2 \pi$-periodic solutions to

$$
u^{\prime \prime}(t)+\nabla G(u)=0 .
$$

Note that from Theorem 4.2 we can immediately deduce an analogous noexistence result valid for the Dirichlet problem associated with $(4.3)$ in $[0, \pi]$, when the potential $G$ is symmetric (and, consequently, $\nabla G$ is an odd function). Indeed, adopting a classic procedure (cf., for instance, [4] and [21]) based on the odd structure of system (4.3), it is possible to establish a correspondence between its odd $2 \pi$-periodic solutions and the solutions of the associated Dirichlet problem 
in $[0, \pi]$. In particular, each solution $u:[0, \pi] \rightarrow \mathbb{R}$ of (4.3) satisfying the Dirichlet boundary conditions

$$
u(0)=0=u(\pi)
$$

is an odd $2 \pi$-periodic solution of (4.3), provided that we extend it to the interval $[-\pi, 0]$ by the oddness relation

$$
u(-t)=-u(t),
$$

and finally to $\mathbb{R}$ by the $2 \pi$-periodicity. Thus, from Theorem 4.2 we infer the following

Corollary 4.3. Assume that there exist $G \in C^{2}\left(\mathbb{R}^{n} ; \mathbb{R}\right)$ with $G(-x)=G(x)$ for every $x \in \mathbb{R}^{n}$, and two constant symmetric matrices $B_{1}, B_{2}$ whose eigenvalues satisfy (4.1). Denote by $H$ the Hessian matrix of $G$, and suppose the validity of (4.2). Then, there exist no nontrivial solutions of (4.3) and (4.4).

We have decided to translate the Lazer's result in a Dirichlet setting, since the theory used in this work is concerned with two-point boundary value problems. Also the definition of index (cf. Definition 1.2) we have adopted refers to Dirichlet problems. We stress the fact that the index introduced in Definition 1.2 is the only one mentioned and employed throughout this paper.

Let us finally remark that from now on the index and the nullity will be calculated with respect to $T=\pi$. According to this notation, it is easy to verify that, given a constant real symmetric matrix $B$, and called $\nu_{1}, \nu_{2}, \ldots, \nu_{n}$ its eigenvalues, its index is given by

$$
i(B)=\sum_{i \in\{1, \ldots, n\}: \nu_{i}>0}\left\lfloor\sqrt{\nu_{i}}\right\rfloor,
$$

where, for every positive $a,\lfloor a\rfloor:=n$ if $n<a \leq n+1$ for some $n \in \mathbb{N}$. Moreover, $\nu(B)$ is the number of positive eigenvalues whose square root belongs to $\mathbb{N}$. (4.6) As a consequence, we observe that the indexes and the nullities of the matrices $B_{1}$ and $B_{2}$ satisfying assumption (4.1) coincide. More precisely,

$$
i\left(B_{1}\right)=i\left(B_{2}\right)=\sum_{i=1}^{n} N_{i},
$$

and $\nu\left(B_{1}\right)=\nu\left(B_{2}\right)=0$.

Following an approach based on the notion of index and its comparison properties recalled in Proposition 2.1, we immediately notice that it is possible to state a generalized version of the Lazer's no-existence result for Dirichlet problems of the form (1.1), where the constant matrices bounds are replaced by paths of symmetric matrices $B_{1}(\cdot), B_{2}(\cdot)$, satisfying $i\left(B_{1}\right)=i\left(B_{2}\right)$. No further conditions on the paths of matrices $B_{j}$ need to be imposed (also in the constant case their respective $i$ th eigenvalues are not necessarily linked each other by the relation (4.1)). In particular, we can state the following theorem. 
Theorem 4.4. Consider $A \in L^{\infty}\left([0, T] \times \mathbb{R}^{n} \times \mathbb{R} ; M_{s}(n)\right)$. Assume the existence of $r_{1}, r_{2} \in \overline{\mathbb{R}}$ with $r_{1}<r_{2}$ and $B_{1}, B_{2} \in L^{\infty}\left([0, T] ; M_{s}(n)\right)$ with

$$
i\left(B_{1}\right)=i\left(B_{2}\right)
$$

such that

$$
B_{1}(\cdot) \leq A(\cdot, x, \lambda)<B_{2}(\cdot) \quad \forall x \in \mathbb{R}^{n}, \forall \lambda \in\left(r_{1}, r_{2}\right) .
$$

Then, problem $(1.1)$ does not admit any solution $(\lambda, u) \in\left(r_{1}, r_{2}\right) \times C^{2}\left([0, T] ; \mathbb{R}^{n}\right)$ with $u \neq \equiv$.

Proof. Let us argue by contradiction, and let $\left(\lambda_{0}, u_{0}\right) \in\left(r_{1}, r_{2}\right) \times C^{2}\left([0, T] ; \mathbb{R}^{n}\right)$ be a solution of (1.1) with $u_{0} \not \equiv 0$. Recalling the Definition 1.2 of nullity and the definition (1.7), we easily infer that

$$
\nu\left(\lambda_{0}, u_{0}\right) \geq 1
$$

(indeed, when $\eta=0$, problem

$$
z^{\prime \prime}(t)+\left(A\left(t, u_{0}(t), \lambda_{0}\right)+\eta \mathrm{Id}\right) z(t)=0
$$

admits the nontrivial solution $u_{0}$, and, consequently, $\eta=0$ is an eigenvalue of (4.10)). Furthermore, by combining assumption (4.8) with Proposition 2.1, we deduce that

$$
i\left(B_{1}\right) \leq i\left(\lambda_{0}, u_{0}\right) \leq i\left(\lambda_{0}, u_{0}\right)+\nu\left(\lambda_{0}, u_{0}\right) \leq i\left(B_{2}\right) .
$$

Hence, according to (4.7), we obtain that $i\left(B_{1}\right)=i\left(\lambda_{0}, u_{0}\right)=i\left(B_{2}\right)$ and $\nu\left(\lambda_{0}, u_{0}\right)=0$, which contradicts (4.9). This completes the proof.

Remark 4.5. The same conclusion of Theorem 4.4 holds if we assume the existence of $r_{1}, r_{2} \in \overline{\mathbb{R}}$ with $r_{1}<r_{2}$ and $B \in L^{\infty}\left([0, T] ; M_{s}(n)\right)$ with $i(B)=0$ such that

$$
A(\cdot, x, \lambda)<B(\cdot) \quad \forall x \in \mathbb{R}^{n}, \forall \lambda \in\left(r_{1}, r_{2}\right) .
$$

We have introduced these no-existence results in order to explain and motivate the context where our assumptions have been chosen. Indeed, the previous theorems provide an example where no bifurcation occurs. Since we are interested in branches of nontrivial solutions bifurcating from $(\lambda, u) \equiv(1,0)$, we clearly need to estimate the nonlinearity by paths of matrices having different indexes. We have noticed that the easiest way to get bifurcation is to require only one jump between the values of the lower bound and the upper bound indexes. The advantage of the one-jump choice is that the index is preserved along the branches of nontrivial solutions (and it is exactly equal to $k$, if the indexes associated with the lower and upper bounds on the nonlinearity are $k$ and $k+1$, respectively) and the eigenspace associated with each solution (according to the definition of nullity) is one-dimensional. 
We conclude this section by presenting a possible application of our main result. In the sequel, we focus on the special case of problem (1.1) where $L+H$ is a constant path of symmetric matrices, defined by

$$
(L+H)(\cdot) \equiv(L+H)=\left[\begin{array}{cc}
a+b & -a \\
-a & a+b
\end{array}\right],
$$

where $a, b>0$. As observed in Remark 2.12, in order to apply our main theorems we need to assume that

$$
\nu(L+H)=1
$$

Being $L+H$ a constant matrix, it is easy to calculate its nullity. In particular, it is immediate to check that its eigenvalues are respectively $b$ and $b+2 a$. Thus, according to (4.6), condition (4.12) is equivalent to require the existence of $k \in \mathbb{N} \cup\{0\}$ such that

$$
\begin{array}{lll}
\text { either } & b+2 a=(k+1)^{2} & \text { and } \quad \sqrt{b} \notin \mathbb{N} \\
\text { or } & b=(k+1)^{2} \quad \text { and } & \sqrt{b+2 a} \notin \mathbb{N} .
\end{array}
$$

Remark 4.6. Note that $i(L+H)=k+[\sqrt{b}]$ if the (4.13) holds, while $i(L+H)=$ $k+[\sqrt{b+2 a}]$ if $(4.14)$ is satisfied, where $[s]$ denotes the integer part of any $s>0$.

Denoting by $\mathbb{R}^{+}$the set of positive real numbers, for each $k \in \mathbb{N} \cup\{0\}$ we define the lines

$$
\begin{aligned}
c_{k} & :=\left\{(a, b) \in \mathbb{R}^{+} \times \mathbb{R}^{+}: b+2 a=(k+1)^{2}\right\}, \\
d_{k}: & =\left\{(a, b) \in \mathbb{R}^{+} \times \mathbb{R}^{+}: b=(k+1)^{2}\right\},
\end{aligned}
$$

along which the nullity of $L+H$ is different from zero (in particular, it is equal to 2 in the intersection points, and it is equal to 1 in the other cases).

Figure 1 illustrates how the values of the index $i(L+H)$ vary in the $a b$ plane. With each region determined by the lines $c_{k}$ and $d_{k}$ we have associated the corresponding index (the number sketched in every region of Fig. 1 is exactly the value of the index). Note that the index of each area is preserved along its right and upper boundaries.

A pair $(a, b)$ of positive coefficients $a$ and $b$ satisfies conditions (4.13) or (4.14) if and only if it lies on the lines $c_{k}$ or $d_{k}$, respectively, but not on their intersection points.

Assume, for instance, that $L+H$ has the form (4.11) where $(a, b) \in c_{k}$ and $n^{2}<b<(n+1)^{2}$. In order to be able to apply Theorem 2.10 , we could estimate from below and above our nonlinearity (whose linearization at $u=0$ is $L+H$ ) respectively by two constant matrices (or paths of matrices) $B_{1}$ and $B_{2}$ of the form (4.11), whose respective pairs of coefficients $\left(a_{1}, b_{1}\right)$ and $\left(a_{2}, b_{2}\right)$ lie in the interior of the blocks bordered by $c_{k}$; in particular $\left(a_{1}, b_{1}\right)$ and $\left(a_{2}, b_{2}\right)$ are respectively situated in the left and right side block.

Analogously, if the pair of coefficients $(a, b)$ of $L+H$ belongs to $d_{k}$ and satisfies $n^{2}<b+2 a<(n+1)^{2}$, then the pairs of coefficients $\left(a_{1}, b_{1}\right)$ and $\left(a_{2}, b_{2}\right)$ 


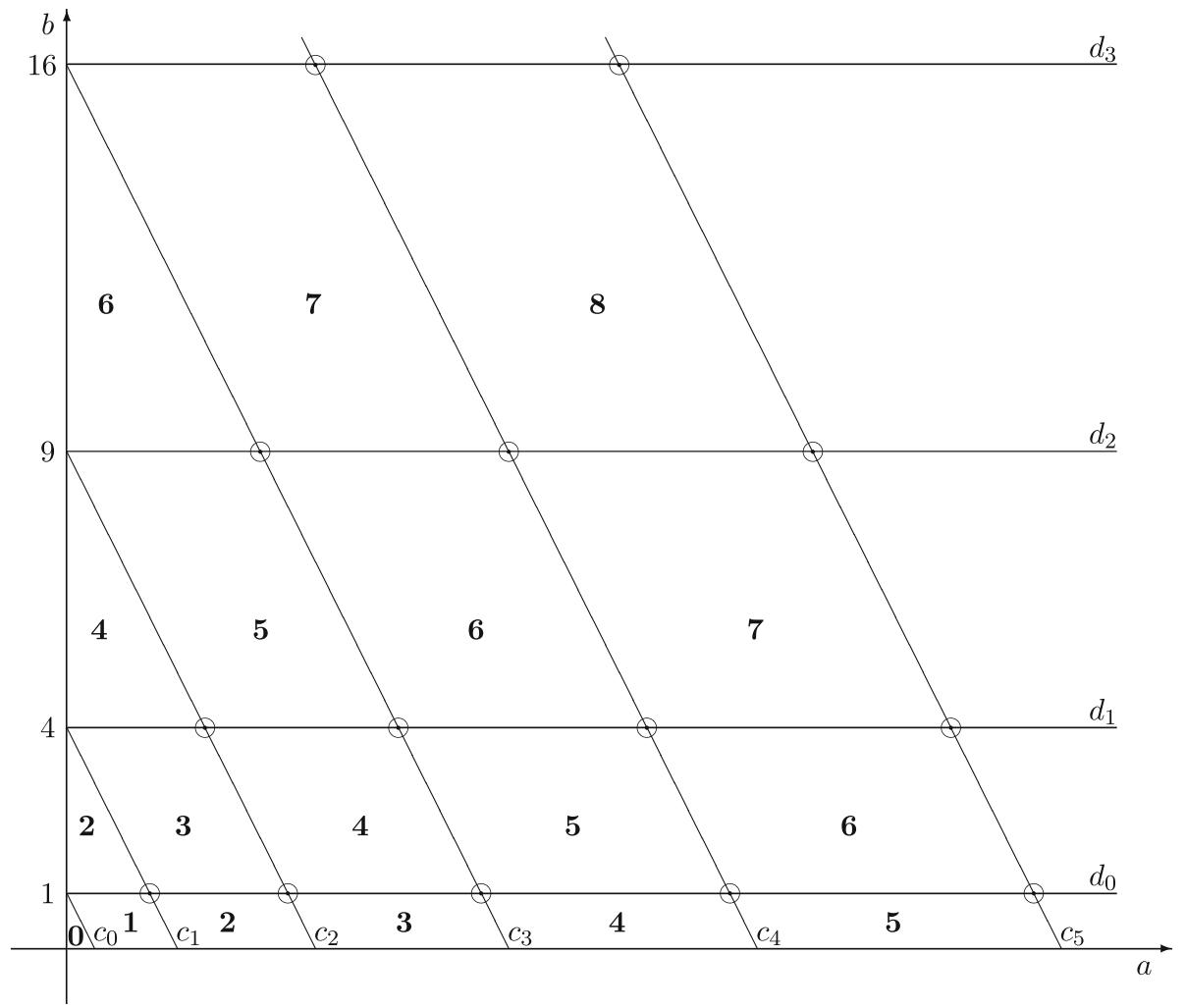

Figure 1. Some non-zero nullity lines with the associated constant index regions

of the bounds $B_{1}$ and $B_{2}$ could be chosen in the regions whose upper and lower border is $d_{k}$, respectively.

Let us analyze a particular case in which the matrix $L+H$ presents the form (4.11). The example we are concerned with is described by the following system

$$
u^{\prime \prime}(t)+\left[\begin{array}{rr}
a & -a \\
-a & a
\end{array}\right] u(t)+\left[\begin{array}{ll}
\left(b+\varepsilon f_{1}(t)\right) & \sin u_{1}(t) \\
\left(b+\varepsilon f_{2}(t)\right) & \sin u_{2}(t)
\end{array}\right]=0,
$$

where $a, b$ are positive constants, $u \equiv\left(u_{1}, u_{2}\right), \varepsilon \in \mathbb{R}$ is suitably small, and

$$
f_{i} \in C(\mathbb{R}, \mathbb{R}) \text { are odd }, 2 \pi \text {-periodic, } \quad f_{i} \neq \equiv, \quad f_{i}(t) \in[0,1] \quad \forall t \in[0, \pi] .
$$

This system represents (with a good approximation) the model of two pendulums coupled by a torsion elastic force, in presence of odd, periodic perturbations of their lengths. We assume that the lengths of the two pendulums are subject to different disturbances. We observe that in case of equal perturbations 
$f_{1}=f_{2}=f$, due to the symmetry of the problem, the set of $2 \pi$-periodic solutions of system (4.15) admits an invariant submanifold constituted by the functions $u$ with equal, $2 \pi$-periodic components $u_{1}=u_{2}=v$, satisfying the scalar equation $v^{\prime \prime}(t)+(b+\varepsilon f(t)) \sin v(t)=0$. Hence, in case of equal perturbations, some particular solutions can be deduced by the study of a scalar equation.

For a detailed mechanical interpretation of problem (4.15) we refer to the final part of Section 5 of the paper [19] by Marlin, dealing with the periodic motion of forced linearly coupled simple pendulums equations. In the literature, many authors have studied existence of periodic solutions for systems of forced pendulum-like equations under the presence of external time-dependent disturbances. In this framework, we wish to mention, among others, the works [9] and [20], from which many other papers have been inspired.

The following theorem provides the existence of bounded branches of $2 \pi$-periodic solutions to system (4.15).

Theorem 4.7. Assume the validity of (4.16). Fix $k \in \mathbb{N} \cup\{0\}$, and suppose that

$$
b+2 a=(k+1)^{2} \quad \text { and } \quad 0<b<1 .
$$

Define

$$
\rho_{1}:=\left\{\begin{array}{ll}
-\pi(1-b) & \text { if } k \neq 1 \\
-\min \left(\pi(1-b), 3-b-\frac{2 b}{\pi}\right) & \text { if } k=1,
\end{array} \quad \rho_{2}:=1-b\right.
$$

Then, there exists a bounded continuum $\mathcal{C} \subset\left(\rho_{1}, \rho_{2}\right) \times C^{2}\left(\mathbb{R} ; \mathbb{R}^{2}\right)$ of solutions $(\varepsilon, u)$ of problem $(4.15)$, odd and $2 \pi$-periodic in the variable $u$, such that $(0,0) \in \mathcal{C}$ and there exists $\left(\varepsilon_{m}, u_{m}\right) \in \mathcal{C}$ satisfying $\varepsilon_{m} \rightarrow \rho_{j}$ with $j \in\{1,2\}$.

Proof. As observed before, due to the odd and periodic structure of our problem, it is possible to provide odd $2 \pi$-periodic solutions to planar system (4.15) from the odd and $2 \pi$-periodic extensions of the solutions to the associated Dirichlet problem in $[0, \pi]$.

For this reason, to prove Theorem 4.7 we can simply study the existence of bounded branches of solutions $(\lambda, u)$ bifurcating from $(1,0)$ to problem

$$
\left\{\begin{array}{l}
u^{\prime \prime}(t)+\left[\begin{array}{rr}
a & -a \\
-a & a
\end{array}\right] u(t)+\left[\begin{array}{ll}
\left(b+(\lambda-1) f_{1}(t)\right) & \sin u_{1}(t) \\
\left(b+(\lambda-1) f_{2}(t)\right) & \sin u_{2}(t)
\end{array}\right]=0 \\
u(0)=0=u(\pi),
\end{array}\right.
$$

where $\varepsilon:=\lambda-1$. We have introduced the variable $\lambda$, since we are interested in treating problem (4.19) with the methods developed in the previous sections. In particular, our aim consists in applying Theorem 2.10, where a bifurcation from the eigenvalue $\lambda=1$ occurs. To this purpose, let us first rewrite our problem in the form (1.1) and (1.2). Notice that the equation in (4.19) can be equivalently 
written in the following form

$$
\begin{aligned}
& u^{\prime \prime}(t)+\left[\begin{array}{cc}
a+b-f_{1}(t) & -a \\
-a & a+b-f_{2}(t)
\end{array}\right] u(t)+\lambda\left[\begin{array}{cc}
f_{1}(t) & 0 \\
0 & f_{2}(t)
\end{array}\right] u(t) \\
& +\left[\begin{array}{c}
\left(b+(\lambda-1) f_{1}(t)\right)\left(\sin u_{1}(t)-u_{1}(t)\right) \\
\left(b+(\lambda-1) f_{2}(t)\right)\left(\sin u_{2}(t)-u_{2}(t)\right)
\end{array}\right]=0 .
\end{aligned}
$$

Thus, if we define

$$
L(t):=\left[\begin{array}{cc}
a+b-f_{1}(t) & -a \\
-a & a+b-f_{2}(t)
\end{array}\right], \quad H(t):=\left[\begin{array}{cc}
f_{1}(t) & 0 \\
0 & f_{2}(t)
\end{array}\right],
$$

and note that $\left(\left(b+(\lambda-1) f_{1}(t)\right)\left(\sin x_{1}-x_{1}\right),\left(b+(\lambda-1) f_{2}(t)\right)\left(\sin x_{2}-x_{2}\right)\right)=$ $o(\|x\|)$, it is easy to conclude that system (4.19) can be represented in the form (1.1) and (1.2).

In order to apply Theorem 2.10 , let us verify the validity of its assumptions.

Notice first that the expression of $L+H$ is exactly the one in (4.11). Observe also that our assumption (4.17) is contained in (4.13), which, in turn, implies that $\nu(L+H)=1$. Hence, condition (1.10) is satisfied.

Moreover, note that $H>0$, since, by definition, $f_{i} \geq 0$ in $[0, \pi]$ and they are not identically zero. Thus, condition (1.11) is verified too.

To prove the validity of the remaining assumptions, we need the following lemma

Lemma 4.8. Consider $\alpha_{i}, \gamma_{i}, \beta \in \mathbb{R}$ with $i \in\{1,2\}$. Then,

$$
\alpha_{1}<\alpha_{2} \quad \text { and } \quad \gamma_{1}<\gamma_{2} \Longrightarrow\left[\begin{array}{cc}
\alpha_{1} & \beta \\
\beta & \gamma_{1}
\end{array}\right]<\left[\begin{array}{cc}
\alpha_{2} & \beta \\
\beta & \gamma_{2}
\end{array}\right] \text {. }
$$

The proof of this lemma simply follows by observing that the difference between the left-hand side and the right-hand side matrices is a diagonal matrix, whose diagonal terms are negative.

Our next aim consists in showing that $\nu(L)=0$.

Recalling (4.16) and the expression of $L$ given in (4.20), from Lemma 4.8 we infer that

$$
M:=\left[\begin{array}{ll}
a+b-1 & -a \\
-a & a+b-1
\end{array}\right]<L(\cdot)<L+H .
$$

By combining Remark 4.6 with assumption (4.17) we immediately get that $i(L+H)=k$.

Moreover, the eigenvalues of $M$ are $b-1$ and $2 a+b-1$. Hence, assumption (4.17) combined with (4.5) leads to $i(M)=k$.

As a consequence, Proposition 2.1 guarantees that $\nu(L)=0$ (and $i(L)=k)$. Thus, also condition (2.11) of Theorem 2.10 is satisfied.

Arguing exactly as above, let us calculate index and nullity of the matrix

$$
\mathcal{A}:=\left[\begin{array}{rr}
a & -a \\
-a & a
\end{array}\right] \text {. }
$$


Asumption (4.17) and Lemma 4.8 ensure that $M<\mathcal{A}<L+H$, whence we infer that

$$
\nu(\mathcal{A})=0 \quad \text { and } \quad i(\mathcal{A})=k .
$$

We are finally interested in proving that our nonlinearity can be estimated by two symmetric matrices having consecutive indexes.

Let us remark that (4.19) can be written in the form (1.1), where

$$
A(t, x, \lambda):=\left[\begin{array}{cc}
a+\left(b+(\lambda-1) f_{1}(t)\right) \frac{\sin x_{1}}{x_{1}} & -a \\
-a & a+\left(b+(\lambda-1) f_{2}(t)\right) \frac{\sin x_{2}}{x_{2}}
\end{array}\right] .
$$

According to the definition of $\rho_{1}$ and $\rho_{2}$ introduced in (4.18), we set

$$
r_{1}:=\rho_{1}+1 \quad \text { and } \quad r_{2}:=\rho_{2}+1 \text {. }
$$

Being $\rho_{1}$ negative and $\rho_{2}$ positive, we immediately deduce that $r_{1}<1<r_{2}$.

Taking into account assumption (4.16) and observing that $\frac{\sin y}{y} \in\left(-\frac{1}{\pi}, 1\right]$, by easy calculations we get that for every $t \in[0, \pi], y \in \mathbb{R}, i \in\{1,2\}$

$$
-\max \left\{-\rho_{1}, \frac{\rho_{2}}{\pi}\right\}<(\lambda-1) f_{i}(t) \frac{\sin y}{y}<\max \left\{\rho_{2},-\frac{\rho_{1}}{\pi}\right\}, \quad \forall \lambda \in\left(r_{1}, r_{2}\right) .
$$

Moreover, from definition (4.18) and recalling that $b<1$, it follows

$$
\max \left\{-\rho_{1}, \frac{\rho_{2}}{\pi}\right\}=-\rho_{1}, \quad \max \left\{\rho_{2},-\frac{\rho_{1}}{\pi}\right\}=\rho_{2}=1-b .
$$

Thus, Lemma 4.8 guarantees that

$$
B_{1} \leq A(\cdot, x, \lambda) \leq B_{2}, \quad \forall x \in \mathbb{R}^{2}, \forall \lambda \in\left(r_{1}, r_{2}\right),
$$

where

$$
B_{1}:=\left[\begin{array}{cc}
a-\frac{b}{\pi}+\rho_{1} & -a \\
-a & a-\frac{b}{\pi}+\rho_{1}
\end{array}\right] \quad \text { and } \quad B_{2}:=\left[\begin{array}{cc}
a+1 & -a \\
-a & a+1
\end{array}\right] .
$$

Let us now calculate their indexes.

The eigenvalues of $B_{2}$ are 1 and $2 a+1$. Furthermore, by assumption (4.17) we infer that

$$
(k+1)^{2}<2 a+1=(k+1)^{2}-b+1<(k+2)^{2},
$$

which, according to (4.5), leads to

$$
i\left(B_{2}\right)=k+1 \text {. }
$$

On the other hand, the eigenvalues of $B_{1}$ are $-\frac{b}{\pi}+\rho_{1}$ and $2 a-\frac{b}{\pi}+\rho_{1}$. Recalling that $\rho_{1}$ is negative and taking into account (4.17), it follows that $2 a-\frac{b}{\pi}+\rho_{1}<$ 
$2 a<(k+1)^{2}$. Moreover, from (4.17) we deduce that

$$
\begin{aligned}
2 a-\frac{b}{\pi}+\rho_{1}= & (k+1)^{2}-b-\frac{b}{\pi}-\pi(1-b)>k^{2}+2 k-\frac{b}{\pi}-\pi(1-b)>k^{2}, \\
& \text { if } k \geq 2, \\
2 a-\frac{b}{\pi}+\rho_{1} \geq & 2 a-\frac{b}{\pi}-3+b+\frac{2 b}{\pi}=1+\frac{b}{\pi}>1=k^{2}, \\
& \text { if } k=1 .
\end{aligned}
$$

By combining the previous inequalities with (4.5), we can finally conclude that

$$
i\left(B_{1}\right)=k \text {. }
$$

Also the hypotheses (2.12) and (2.13) of Theorem 2.10 are satisfied. Hence, we can apply it to deduce the existence of a continuum $\widetilde{\mathcal{C}} \subset\left(r_{1}, r_{2}\right) \times C^{2}\left([0, \pi] ; \mathbb{R}^{2}\right)$ of solutions of problem $(4.19)$, containing $(1,0)$ and such that there exists $\left(\lambda_{m}, u_{m}\right) \in \widetilde{\mathcal{C}}$ satisfying

$$
\text { either } \quad\left\|u_{m}\right\| \rightarrow+\infty \quad \text { or } \quad \lambda_{m} \rightarrow r_{j} \quad \text { with } j \in\{1,2\} .
$$

Moreover, $\widetilde{\mathcal{C}} \backslash\{(1,0)\}$ is composed by nontrivial solutions $(\lambda, u)$ of problem (4.19) with $\nu(\lambda, u)=1$ and $i(\lambda, u)=k$.

To complete our proof, it remains to prove the following claim, which ensures the boundedness of the continuum.

Claim. The continuum $\widetilde{\mathcal{C}}$ is bounded in the variable $u$.

Proof of the claim. Let us now argue by contradiction, assuming the existence of $\left(\lambda_{m}, u_{m}\right) \in \widetilde{\mathcal{C}}$ such that $\left\|u_{m}\right\| \rightarrow+\infty$ as $m \rightarrow+\infty$. Let us first divide problem (4.19) by $\left\|u_{m}\right\|$. Defining $z_{m}:=\frac{u_{m}}{\left\|u_{m}\right\|}$ and $u_{m}:=\left(u_{m, 1}, u_{m, 2}\right)$, we obtain

$$
\left\{\begin{array}{l}
z_{m}^{\prime \prime}(t)+\left[\begin{array}{rr}
a & -a \\
-a & a
\end{array}\right] z_{m}(t)+\left[\begin{array}{l}
\left(b+\left(\lambda_{m}-1\right) f_{1}(t)\right) \frac{\sin u_{m, 1}(t)}{\left\|u_{m}\right\|} \\
\left(b+\left(\lambda_{m}-1\right) f_{2}(t)\right) \frac{\sin u_{m, 2}(t)}{\left\|u_{m}\right\|}
\end{array}\right]=0, \\
z_{m}(0)=0=z_{m}(\pi) .
\end{array}\right.
$$

Since $\lambda_{m} \in\left(r_{1}, r_{2}\right), f_{i}(t) \in[0,1]$ for every $t \in[0, \pi]$, and $\lim _{m \rightarrow+\infty}\left\|u_{m}\right\|=+\infty$, we immediately infer that the last addendum in system (4.24) tends to zero in $C\left([0, \pi], \mathbb{R}^{2}\right)$ as $m \rightarrow+\infty$. Moreover, $\lambda_{m}$ and $z_{m}$ admit subsequences (still denoted, respectively, with $\lambda_{m}$ and $z_{m}$ ) such that

$$
\begin{aligned}
& \lim _{m \rightarrow+\infty} \lambda_{m}=\lambda_{0} \in\left[r_{1}, r_{2}\right], \\
& z_{m} \rightarrow z_{0} \text { in } C\left([0, \pi], \mathbb{R}^{2}\right) \text { and } z_{m} \rightarrow z_{0} \text { in } H_{0}^{1}\left([0, \pi] ; \mathbb{R}^{2}\right) .
\end{aligned}
$$

Recalling the definition of $\mathcal{A}$ in (4.21), integrating (4.24) and then, passing to the limit, we deduce that

$$
z_{0}^{\prime \prime}(t)+\mathcal{A} z_{0}(t)=0, \quad z_{0}(0)=0=z_{0}(\pi) .
$$


Since $\left\|z_{0}\right\|=\left\|z_{m}\right\|=1$, we conclude that $z_{0}$ is a nontrivial solution of (4.25), in contradiction with (4.22). The proof of the claim is complete.

We have so proved the existence of a bounded continuum $\widetilde{\mathcal{C}} \subset\left(r_{1}, r_{2}\right) \times C^{2}$ $\left([0, \pi] ; \mathbb{R}^{2}\right)$ of solutions of problem $(4.19)$, containing $(1,0)$ and such that there exists $\left(\lambda_{m}, u_{m}\right) \in \widetilde{\mathcal{C}}$ satisfying $\lambda_{m} \rightarrow r_{j}$ with $j \in\{1,2\}$. We also know that $\nu(\lambda, u)=1$ and $i(\lambda, u)=k$, for every $(\lambda, u) \in \widetilde{\mathcal{C}} \backslash\{(1,0)\}$.

Extending the solutions of the Dirichlet problem (4.19) by oddness and $2 \pi$ periodicity, and recalling that $\varepsilon=\lambda-1, \rho_{i}=r_{i}-1$ for each $i \in\{1,2\}$, we achieve the thesis.

We finally present a result which is complementary to Theorem 4.7. Indeed, while in Theorem 4.7 we assume the validity of (4.13) for $b<1$, in the following theorem we require the validity of a particular case of (4.14).

Theorem 4.9. Assume the validity of (4.16) and suppose that

$$
b=1 \quad \text { and } \quad \frac{k^{2} \pi+1}{2 \pi}<a<\frac{k^{2}+2 k}{2}, \quad k \in \mathbb{N} .
$$

Let $\delta$ be a small positive constant such that $2 a-k^{2}-\frac{1}{\pi}-\delta>0$. Define

$$
\rho_{1}:=-\min (3 \pi, \xi \pi, \gamma), \quad \rho_{2}:=\min (3, \xi, \gamma \pi)
$$

where

$$
\gamma:=2 a-k^{2}-\frac{1}{\pi}-\delta \quad \text { and } \quad \xi:=k^{2}+2 k-2 a
$$

Then, the same conclusion of Theorem 4.7 follows.

The proof of this theorem follows precisely the same steps of the one of Theorem 4.7, and therefore it is omitted. We conclude the work by briefly comparing the alternative between (4.17) and (4.26) under which we obtain global branches of odd $2 \pi$-periodic solutions for (4.15) with the general alternative between (4.13) and (4.14) ensuring that $(\varepsilon, u)=(0,0)$ is a bifurcation point. Observe that we are allowed to provide global bifurcation results only for small values of the variable $b$ (which should be chosen in the interval $(0,1]$ ), since this variable belongs to the nonlinear term of our system and, according to (4.23), it is the coefficient of the changing sign functions $\frac{\sin u_{i}}{u_{i}}$. 


\section{Acknowledgments}

The author is very grateful to Prof. Rafael Ortega for suggestions on the subject of the paper and for his precious advices. The author wishes also to thank Prof. Anna Capietto for very useful discussions. Finally, we would thank the referee for the valuable comments.

\section{References}

[1] Ahmad, S.: An existence theorem for periodically perturbed conservative systems. Michigan Math. J. 20, 385-392 (1973)

[2] Capietto, A., Dambrosio, W.: Preservation of the Maslov index along bifurcating branches of solutions of first order systems in $\mathbb{R}^{N}$. J. Differ. Equ. 227, 692-713 (2006)

[3] Capietto, A., Dambrosio, W., Papini, D.: Detecting multiplicity for systems of second-order equations: an alternative approach. Adv. Differ. Equ. 10, 553$578(2005)$

[4] Castro, A., Lazer, A.C.: On periodic solutions of weakly coupled systems of differential equations. Boll. Un. Mat. Ital. B 18, 733-742 (1981)

[5] Chang, K.C.: Principal eigenvalue for weight matrix in elliptic systems. Nonlinear Anal. 46, 419-433 (2001)

[6] Dalbono, F., Rebelo, C.: Multiplicity of solutions of Dirichlet problems associated with second order equations in $\mathbb{R}^{2}$. Proc. Edinb. Math. Soc. (2) (2009)

[7] de Figueiredo, D.: Positive solutions of semilinear elliptic problems. Differential Equations. In: de Figueiredo, D.G., Honig, C.S. (eds.) Proceedings São Paulo, 1981, pp. 34-87. Lecture Notes in Mathematics, vol. 957. Springer, Berlin (1982)

[8] Dong, Y.: Index theory, nontrivial solutions, and asymptotically linear second-order Hamiltonian systems. J. Differ. Equ. 214, 233-255 (2005)

[9] Drábek, P., Invernizzi, S.: Periodic solutions for systems of forced coupled pendulumlike equations. J. Differ. Equ. 70, 390-402 (1987)

[10] Fleckinger, J., Pardo, R.: Bifurcation for an elliptic system coupled in the linear part. Nonlinear Anal. 37, 13-30 (1999)

[11] Fleckinger, J., Pardo, R., de Thélin, F.: Four-parameter bifurcation for a $p$ Laplacian system. Electron. J. Differ. Equ. 6, 1-15 (2001)

[12] Furi, M., Spadini, M.: Branches of forced oscillations for periodically perturbed second order autonomous ODEs on manifolds. J. Differ. Equ. 154, 96-106 (1999)

[13] García-Huidobro, M., Manásevich, R., Zanolin, F.: A Fredholm-like result for strongly nonlinear second order ODE's. J. Differ. Equ. 114, 132-167 (1994) 
[14] Hartman, P.: Ordinary Differential Equations, 2nd edn. Birkhäuser, Boston (1982)

[15] Hess, P.: On the eigenvalue problem for weakly coupled elliptic systems. Arch. Ration. Mech. Anal. 81, 151-159 (1983)

[16] Krasnosel'skii, M.A., Burd, V.S., Kolesov, Y.S.: Nonlinear Almost Periodic Oscillations. Wiley, New York (1973)

[17] Lazer, A.C.: Applications of a lemma on bilinear forms to a problem in nonlinear oscillations. Proc. Am. Math. Soc. 33, 89-94 (1972)

[18] Lazer, A.C., Sánchez, D.A.: On periodically perturbed conservative systems. Michigan Math. J. 16, 193-200 (1969)

[19] Marlin, J.A.: Periodic motions of coupled simple pendulums with periodic disturbances. Int. J. Non-Linear Mech. 3, 439-447 (1968)

[20] Mawhin, J.: Forced second order conservative systems with periodic nonlinearity. Ann. Inst. H. Poincaré Anal. Non Linéaire 6, suppl., 415-434 (1989)

[21] Mawhin, J., Willem, M.: Variational methods and boundary value problems for vector second order differential equations and applications to the pendulum equation. Nonlinear analysis and optimization (Bologna, 1982), pp. 181-192. Lecture Notes in Math., vol. 1107. Springer, Berlin (1984)

[22] Papageorgiou, N.S., Staicu, V.: Multiple solutions for strongly resonant periodic systems. Nonlinear Anal. 67, 1895-1907 (2007)

[23] Rabinowitz, P.H.: Some global results for nonlinear eigenvalue problems. J. Funct. Anal. 7, 487-513 (1971)

[24] Stavrakakis, N.M., Zographopoulos, N.B.: Bifurcation results for quasilinear elliptic systems. Adv. Differ. Equ. 8, 315-336 (2003)

[25] Ward, J.R.: Periodic solutions of perturbed conservative systems. Proc. Am. Math. Soc. 72, 281-285 (1978)

[26] Zhang, G.Q., Liu, X.P., Liu, S.Y.: Remarks on a class of quasilinear elliptic systems involving the $(p, q)$-Laplacian. Electron. J. Differ. Equ. 20, 1-10 (2005)

F. Dalbono

Dipartimento di Matematica, Università di Torino

Via Carlo Alberto 10, 10123 Turin, Italy

e-mail: francesca.dalbono@unito.it

Received: 25 June 2008

Accepted: 15 April 2009 